\title{
Can dynamic magnetic resonance imaging replace static magnetic resonance sequences in evaluation of temporomandibular joint dysfunction?
}

\section{Zeinab Sobhy Ahmed Sayed Ahmed Kamel, Mohammad Hassan Rashad El-Shafey, Omar Ahmed Hassanien and} Hanan Ahmad Nagy*

\begin{abstract}
Background: Static MRI was used as an effective tool for diagnosis of temporomandibular joint dysfunction instead of invasive techniques such as arthroscopy and arthrography. The purpose of this study was to detect whether dynamic MRI can be used instead of static MRI in diagnosis of TMJ dysfunction or not.

Results: According to disc displacement, anterior disc displacement was detected in 29 joints (36.25\%) by both static and dynamic MRIs, and medial and lateral disc displacements were detected only by static MRI. Regarding disc mobility, dynamic MRI detects stuck disc in 4 joints versus 2 joints detected by static MRI ( $p$ value 0.008). Condylar translation was abnormal in 18 joints (22.5\%) by static MRI and in 26 joints (32.5\%) by dynamic MRI ( $p$ value $<0.001$ ). The detection rate of articular disc for dynamic MRI was $87.5 \%$ versus static MRI $92.5 \%$ ( $p$ value 0.038), and the detection rate of condylar head for dynamic MRI was $97.5 \%$ versus static MRI $100 \%$ ( $p$ value 0.012 ).

Conclusion: Dynamic MRI cannot replace static MRI in evaluation of TMJ dysfunction; both of them support each other for accurate diagnosis and better image quality.
\end{abstract}

Keywords: Temporomandibular joint, Static MRI, Dynamic MRI, Disc displacement

\section{Background}

The temporomandibular joint (TMJ) is one of the most complex joints of the body. It lies between the jaw and the skull, more particularly the mandibular condylar process with the temporal bone, with an articular disc in the space between them. It is one of the important structures aiding in the mouth opening and closing during articulation, chewing, and swallowing $[1,2]$.

TMJ disorder is a common problem and affects up to one third of all adults at some stage in their life. It refers to impaired functioning of the TMJ and frequently

\footnotetext{
* Correspondence: hanan.nagy84@hotmail.com

Radiodiagnosis \& Medical Imaging Department, Faculty of Medicine, Tanta University, El-geish Street, Tanta, Gharbya Governorate, Egypt
}

involves an abnormal disc-condyle relationship and pain $[2,3]$.

Clinical symptoms of temporomandibular joint disorders (TMD), including pain, decreased mandibular movement, and mastication problems, can also occur in non-TMJ disorders [4].

The most frequent cause of TMJ dysfunction is internal derangement which refers to an alteration in the normal pathways of motion of the TMJ that largely involves the function of the articular disc; therefore, these alterations have been also referred to as disc derangement [2]. The main dysfunctional aspect of disc physiology is disc displacement that may be caused by trauma, laxity of ligaments, bruxism, synovial fluid alteration, and improper activity of lateral pterygoid muscles [5]. 
Disc displacement can be classified according to the relation of the displaced disc with mandibular condyle into anterior, lateral, medial, posterior, and rotational displacement which includes anterolateral and anteromedial displacement. It may be disc displacement with reduction or disc displacement without reduction [6].

Imaging plays a key role in delineating the anatomic changes of the TMJ, assisting in identifying the type of TMD, assessing treatment response, providing therapeutic intervention, and guiding surgical management [4].

The functional dynamics of TMJ internal derangement were optimally evaluated by the invasive arthrographic technique in the late 1970s to late 1980s. This technique was difficult to perform and painful to the patient [7].

With the advent of noninvasive imaging techniques such as CT, the arthrographic technique has been generally desolated. However, the obvious limitation of the noninvasive $\mathrm{CT}$ is the lack of dynamic imaging as well as the relatively poor anatomic resolution and the high radiation dose to the lens of the eye [5].

Currently, magnetic resonance imaging (MRI) with its high soft tissue recognition plays an effective role in diagnosing the temporomandibular joint disorders. It overcomes conventional radiography, conventional tomography, and computerized tomography by its ability to show neat soft tissue contrast and characterize the structures of temporomandibular joint. It can also distinguish internal anatomical features of the joint to a degree that no other imaging procedure can match [8].

The diagnosis of TMD usually includes conventional MRI using several static positions of the closed and opened mouth. At these days, some investigators suggested a number of fast pulse sequences for dynamic MRI [9].

The aim of our study was to assess the dynamic MRI as a diagnostic tool for diagnosis of temporomandibular joint dysfunction and its ability to diagnose different types of displacement in comparison to static MRI.

\section{Methods}

\section{Study population}

This prospective study was conducted on 40 patients clinically diagnosed as temporomandibular joint dysfunction. The age of the selected patients ranged from 18 to 66 years with a mean of 29.3 years. They were referred from orthopedic, physiotherapy, or oral and maxillofacial surgery departments to MRI unit in

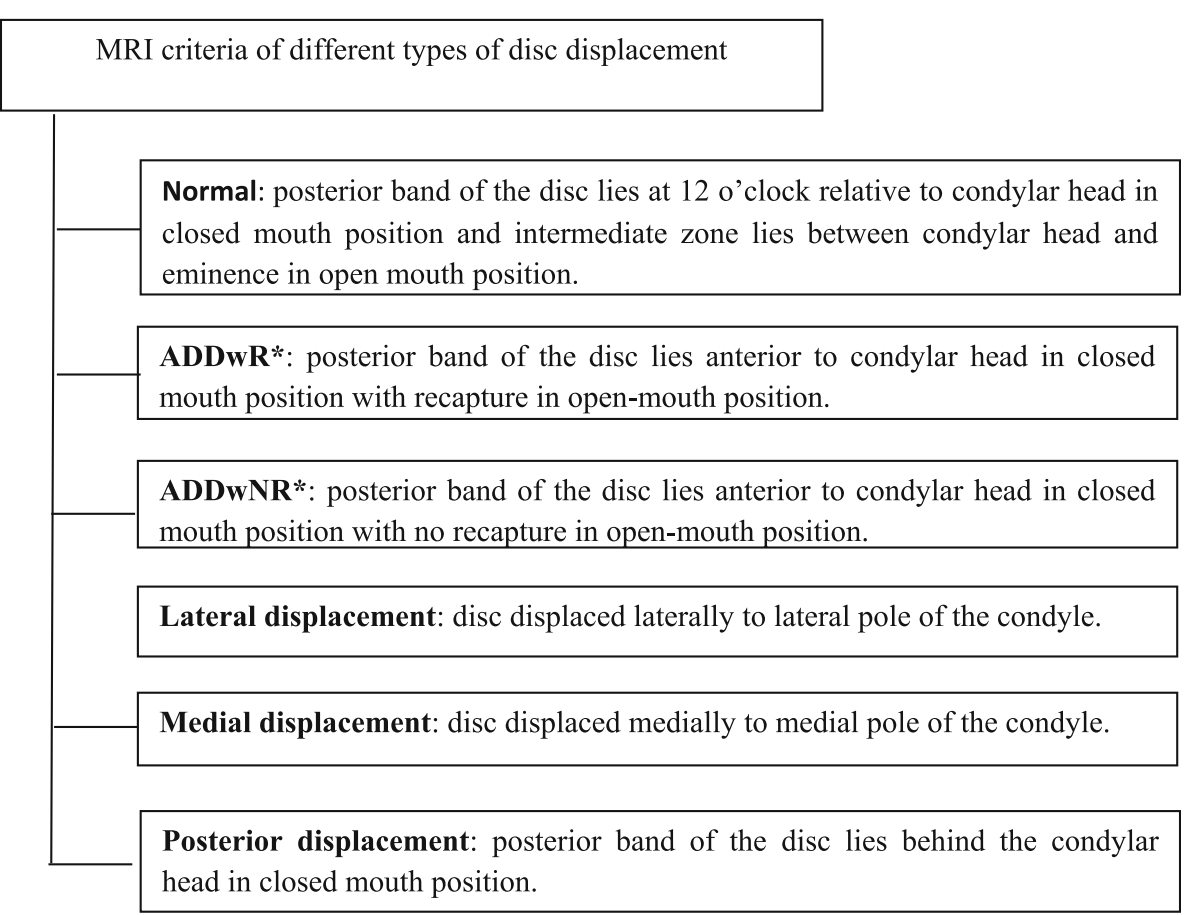

*ADDwR: Anterior disc displacement with reduction

*ADDwNR: Anterior disc displacement with no reduction

Fig. 1 MRI criteria of different types of TMJ disc displacement. *ADDwR, anterior disc displacement with reduction. *ADDwNR, anterior disc displacement with no reduction 


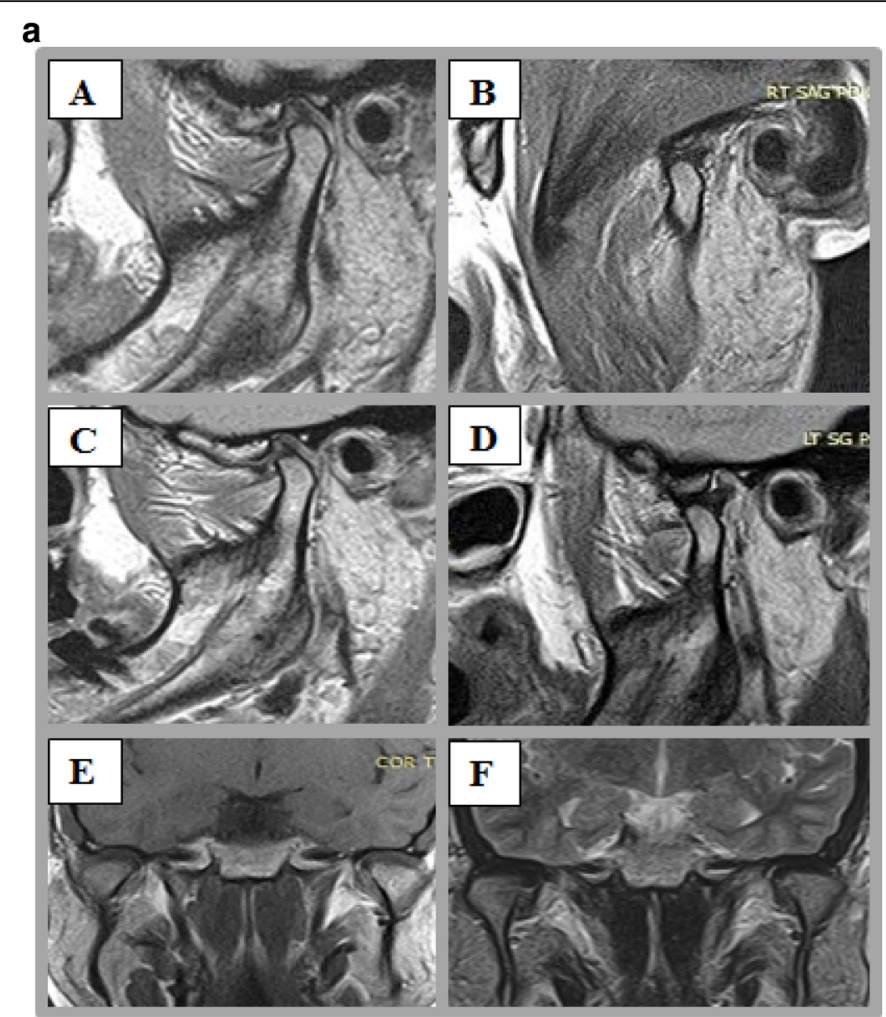

\section{b}
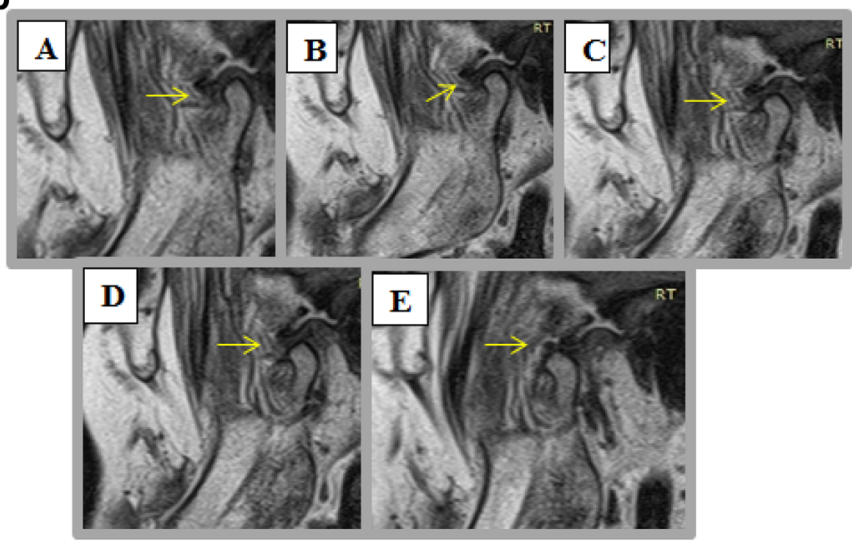

。

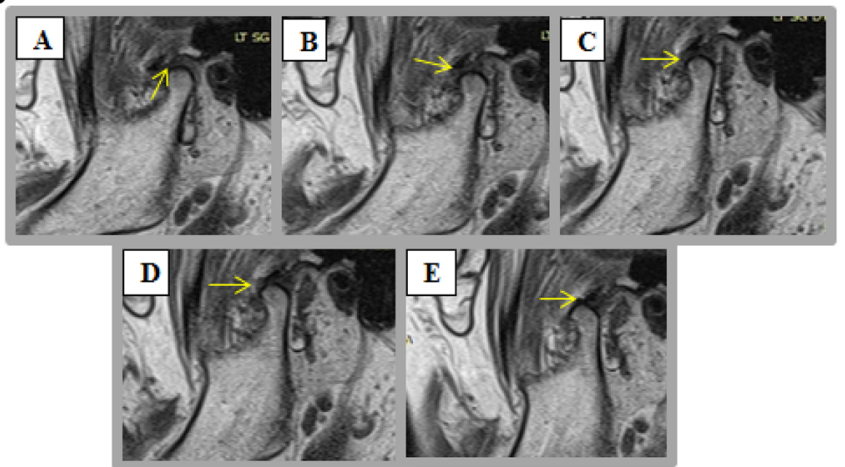

Fig. 2 (See legend on next page.) 
(See figure on previous page.)

Fig. 2 a (A-F) A 43 year old female patient, presented clinically by bilateral temporomandibular joint pain. Sagittal PDWI MRI of both TMJs in closed position ( $A, C$ ) and in open position (B, D) and coronal T1 and T2 WI MRI (E, F) revealed normal shape and position of articular disc in both closed and open positions with no sideway displacement. No effusion or degenerative changes. b (A-E) Dynamic MRI study of right TMJ revealed normal synchronized movement of the articular disc (arrow) with hypermobile condylar translation. c (A-E) Dynamic MRI study of left TMJ revealed normal synchronized movement of the articular disc (arrow) with hypermobile condylar translation

a

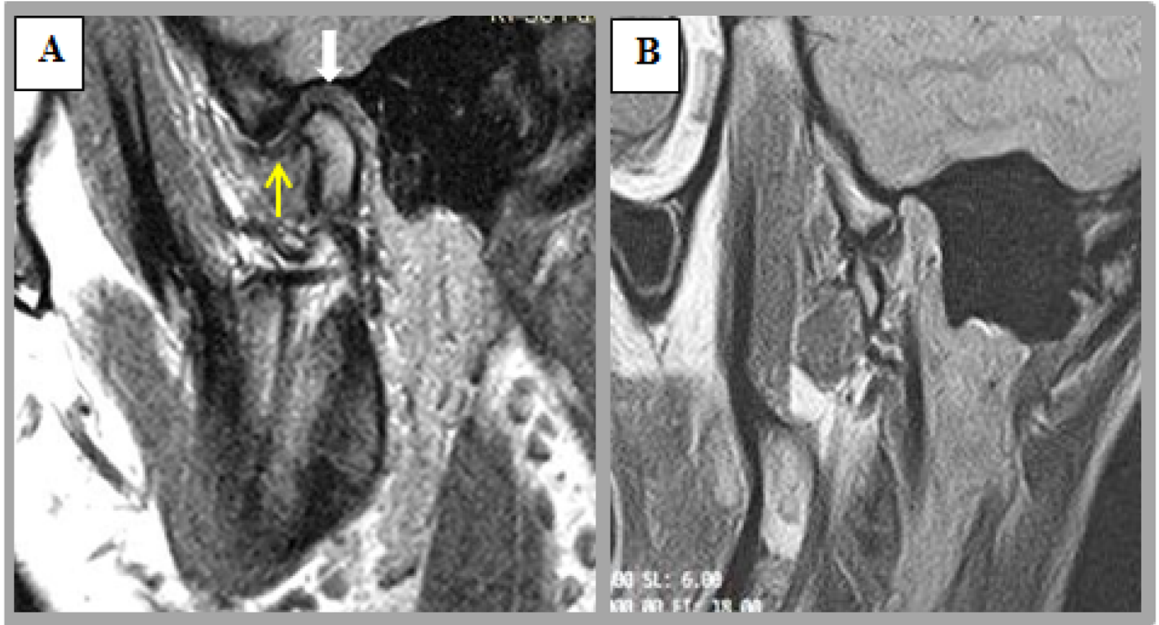

b
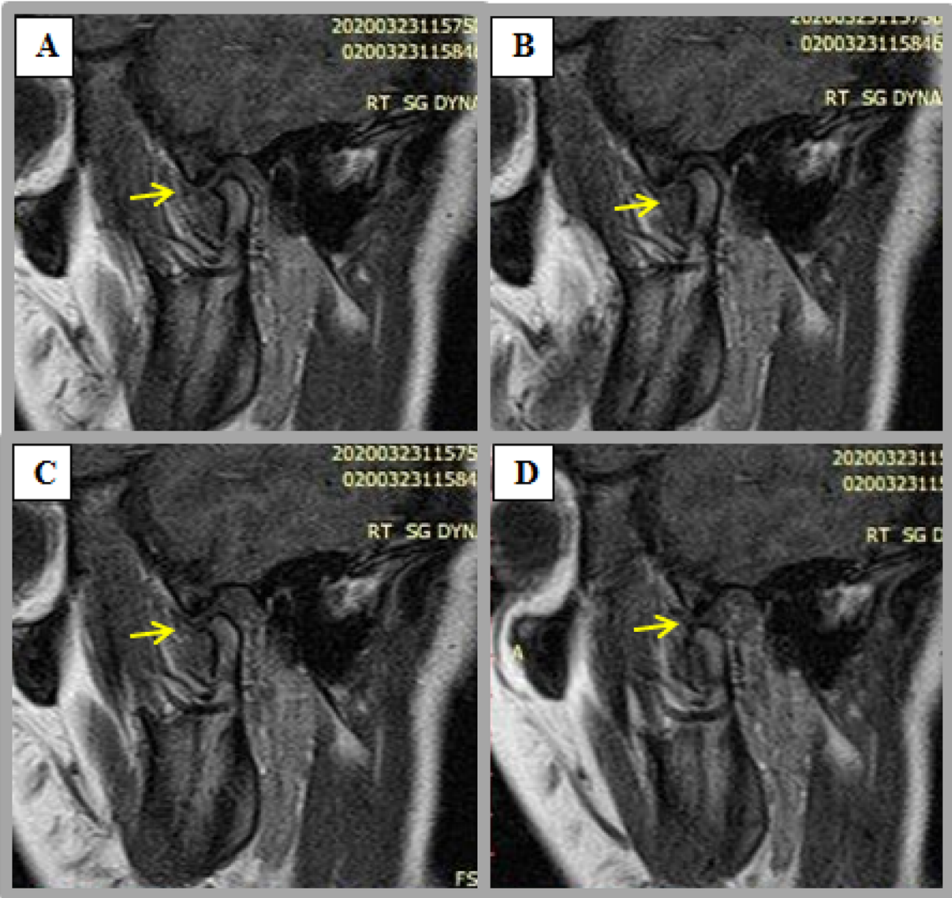

Fig. 3 a (A, B) A 21-year-old female patient, presented clinically by temporomandibular joint pain and clicking on the right side. Sagittal PDWI MRI of right TMJ in closed (A) and open (B) mouth position revealed anterior disc displacement with reduction: posterior band of articular disc (yellow arrow) is seen anterior to the condyle in closed mouth view with normal position in open view. Thickening of superior layer of retrodiscal tissue was noted giving pseudodisc sign (white arrow). b (A-D) Dynamic MRI study of right TMJ revealed anteriorly displaced disc in relation to condylar head with reduction on opening of the mouth associated with normal condylar translation 

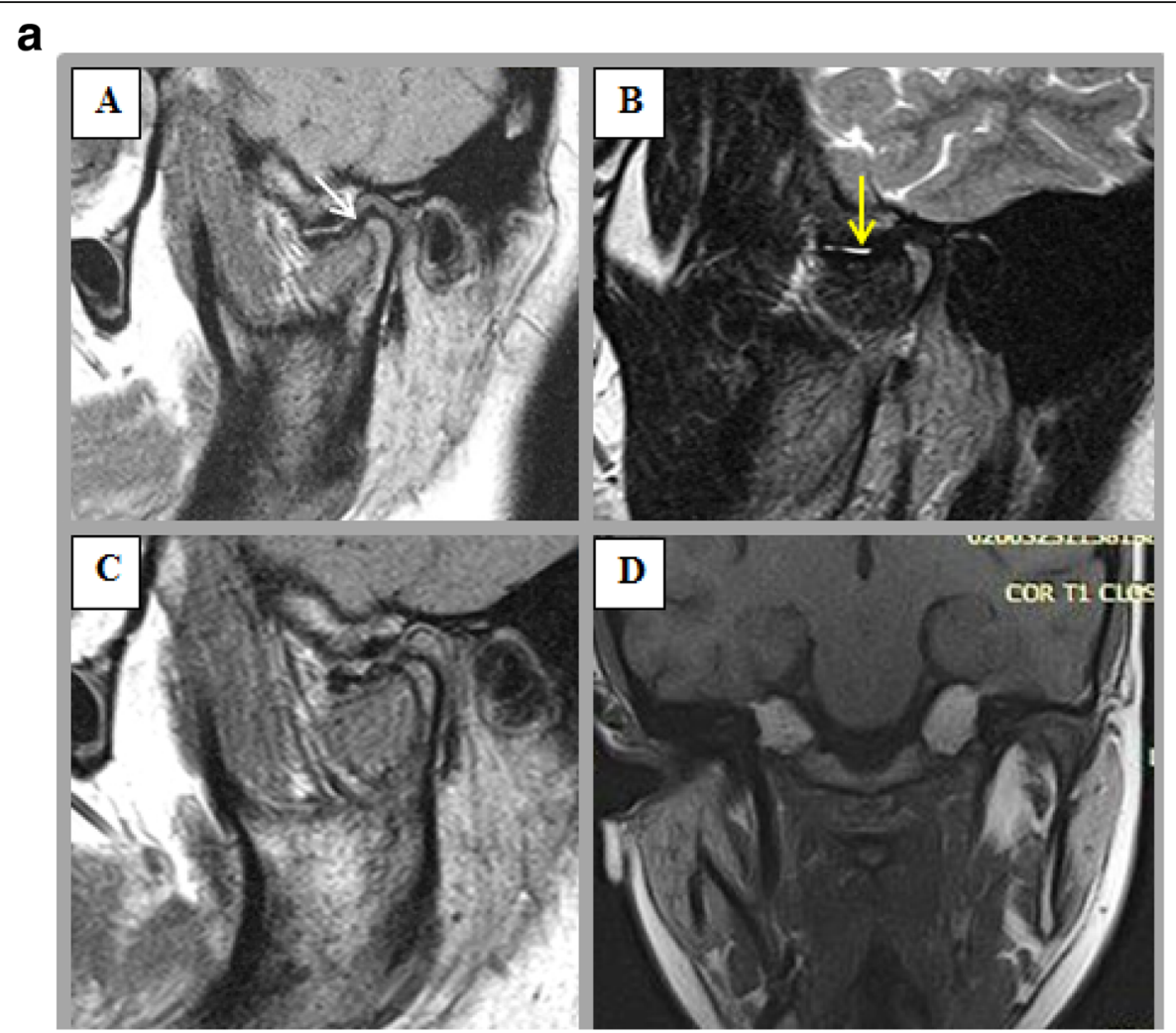

b
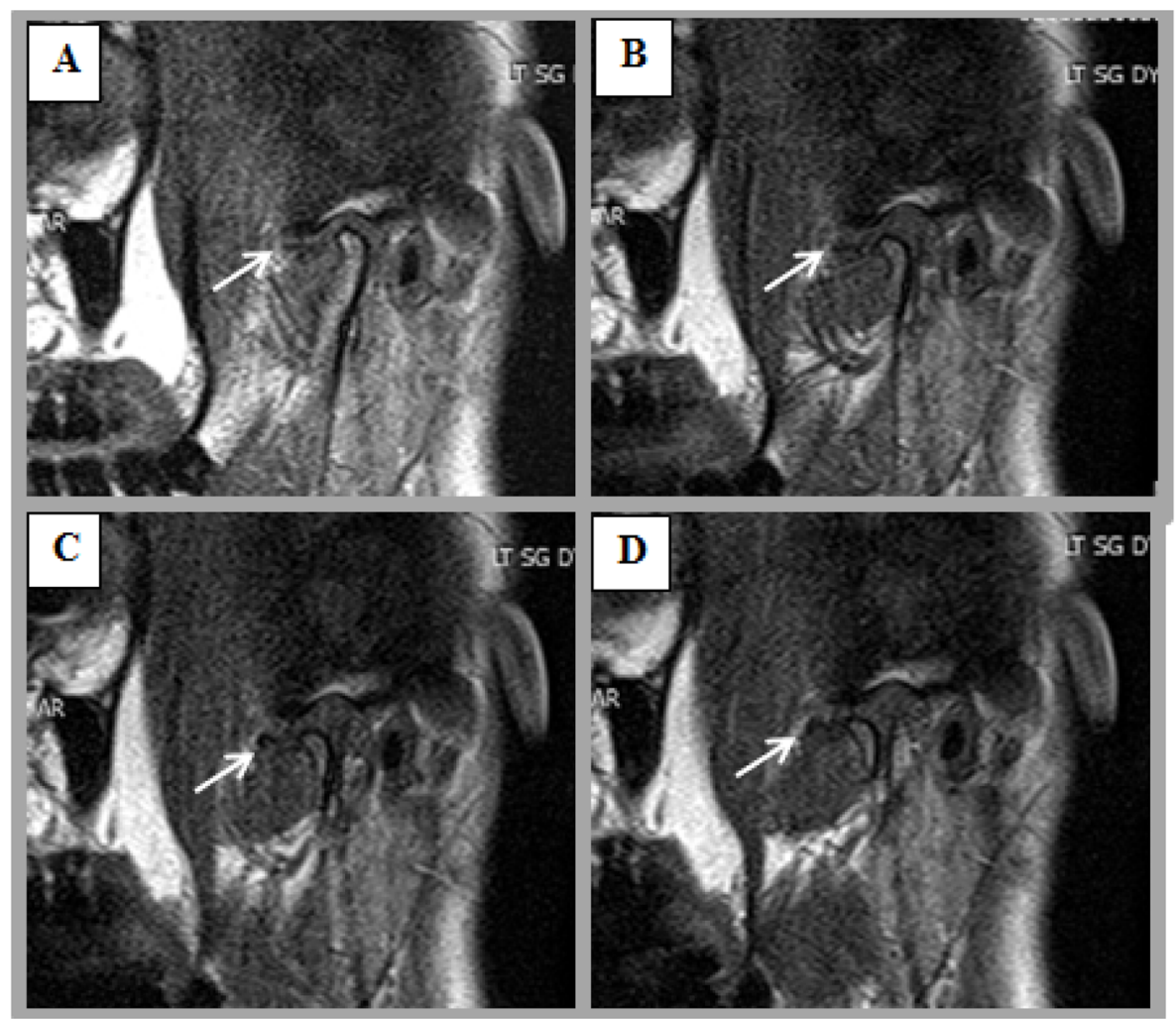

Fig. 4 (See legend on next page.) 
(See figure on previous page.)

Fig. 4 a (A-D) A 25-year-old female patient, presented clinically by temporomandibular joint pain and limitation of movement on the left side. Sagittal PDWI (A) and T2WI (B) MRI of left TMJ in closed and open (C) mouth position and coronal T1 (D) revealed anteriorly displaced deformed disc (flattened) seen in closed mouth position with no reduction in open mouth position associated with joint effusion (yellow arrow) and thickening of retrodiscal tissue "pseudodisc"(white arrow). No side way displacement. b (A-D) Dynamic MRI study of left TMJ revealed anteriorly displaced disc in relation to condylar head without reduction on opening of the mouth associated with limited condylar translation

radiodiagnosis department over a period of 1 year from January 2019 to January 2020.

Approval of Research Ethics Committee (REC) and informed consent were obtained from all participants in this study after explanation of the benefits and risks of the procedure. Privacy and confidentiality of all patients' data were guaranteed. All data provisions were monitored and used for scientific purpose only.

The included criteria were patients with chronic temporomandibular joint pain and dysfunction (more than 1 month) as diagnosed clinically. There is no gender predilection.

Exclusion criteria were patients with previous TMJ or face surgery, or previous facial bone fracture; patients with contraindications to MRI examination such as patients with any metallic prosthesis or artificial pacemakers; or claustrophobic patients or uncooperative patients with mental and behavioral disorders.

\section{All the included participants were subjected to magnetic resonance imaging}

All MRI scans were performed using a 1.5-T GE (General Electric) machine.

\section{Patient preparation}

- The patients were instructed to remove all metallic objects such as hairpins, coins, or ear rings.

- The procedure was explained for reassurance, and the patients were informed about the length of the examination and the value of remaining motionless.

- The patients were trained on slowly opening and closing the mouth for the dynamic study.

\section{Patient position}

All patients were examined in supine position with both arms adducted using the special TMJ dual coil.

\section{MRI protocol}

Imaging started by obtaining localizer images in the axial planes passing through the two TMJs. Image acquisitions in the sagittal and coronal planes were planned in these axial planes.

Conventional static MRI The static images were acquired in the closed and open mouth positions; variable sized sterilized syringes put between upper and lower teeth (used as a bite block) to stabilize the patient's jaw in the open mouth position. Images were obtained on oblique sagittal T1-weighted fast-spin-echo sequence; T2-weighted fast-spin-echo sequence, proton density fat-suppression sequences (PD), and gradient sequences with oblique coronal views done in $\mathrm{T} 1$ - and $\mathrm{T} 2$ weighted images (in closed position). Matrix is $256 \times$ 128 , and field of view is $10-12 \mathrm{~cm}$. The number of slices is 18 , and slice thickness/space is $3 \mathrm{~mm}$.

- TMJ localizer: plane, axial T1; number of slices, 10; slice thickness/space, 5/2 mm; TR/TE, 300/12 ms.

- Sagittal and coronal oblique T1-weighted image TR/ TE, 400-500/10-20 m s.

- Sagittal and coronal oblique T2-weighted image TR/ TE, 2600/120 m s.

- Sagittal oblique PD weighted image TR/TE, 2000/ $10-14 \mathrm{~m} \mathrm{~s}$.

- Sagittal oblique GRE image TR/TE, 600/15 m s.

Dynamic MRI It was obtained as rapid acquisition of static images using a single shot fast spin echo (SSFSE proton density sequence) during slowly opening and closing of the mouth. Eight to ten images were obtained. These images were displayed sequentially as a cine loop.

Dynamic imaging was performed in straight sagittal orientation along the predictable pathway of condylar motion.

The sequence parameters were TR/TE, 88/12 $\mathrm{m} \mathrm{s}$; slice thickness/gap, 3.5/0.6 mm; FOV, $15 \mathrm{~cm}$; and matrix, 256 $\times 160$.

Also, SSFSE gradient echo sequence was performed in some cases with the following parameters: TR/TE, 90/7 $\mathrm{m} \mathrm{s}$; slice thickness/gap, 5/0.6 mm; FOV, $15 \mathrm{~cm}$; and matrix, $320 \times 192$.

\section{Image analysis}

- All the static and dynamic MR images of all patients were analyzed by two radiologists with 20 and 9 years of TMJ MRI experience, blinded to the clinical data and laboratory indicators, in a standard clinical Picture Archiving and Diagnostic System workstation, and final decisions reached by consensus are reported. 

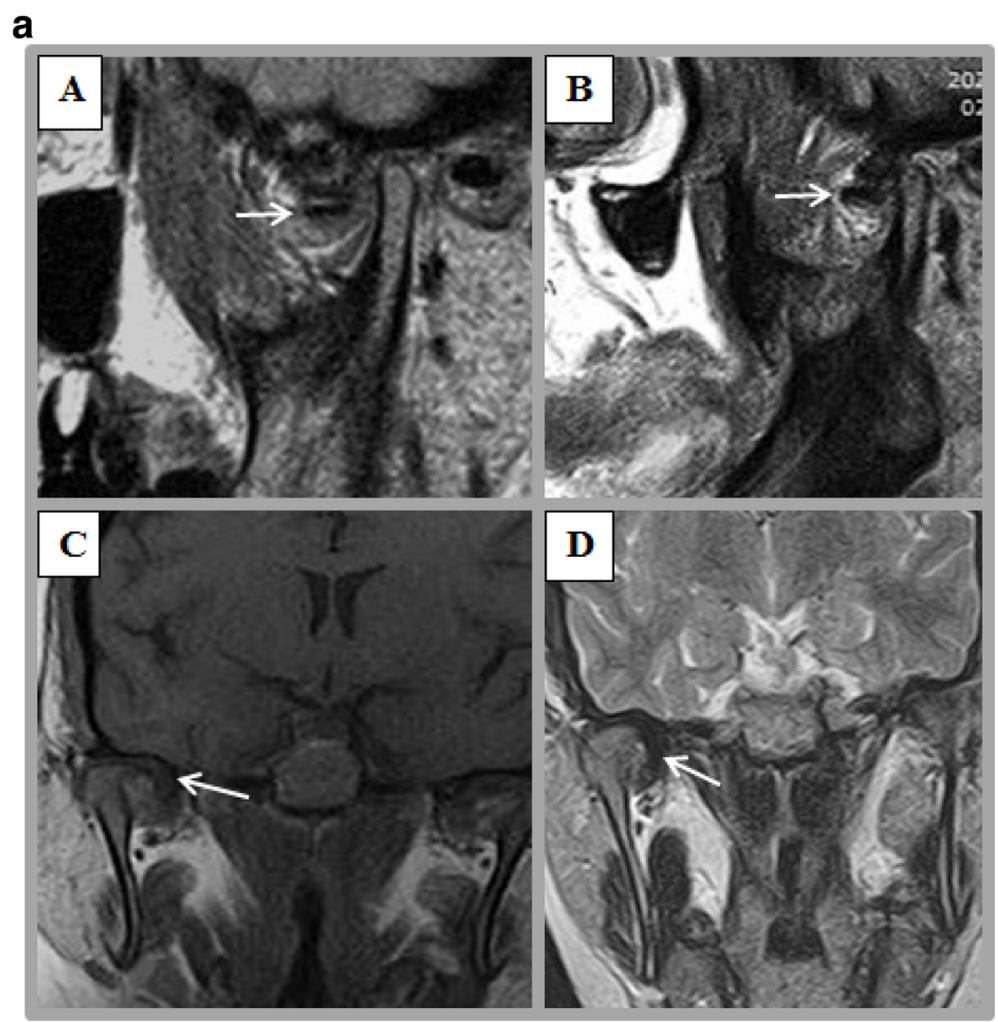

b
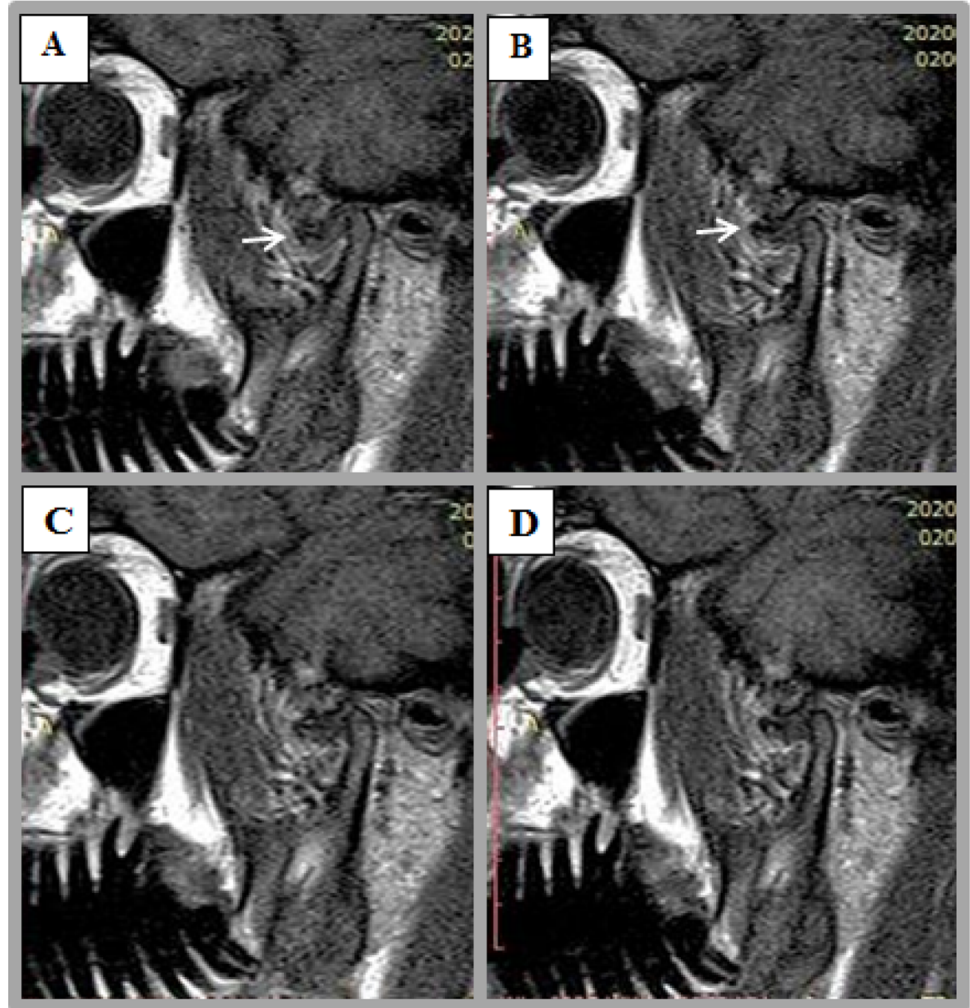

Fig. 5 (See legend on next page.) 
(See figure on previous page.)

Fig. 5 a (A-D) A 20-year-old male patient presented clinically by temporomandibular joint pain and limitation of movement on the right side. Sagittal PDWI MRI of right TMJ in closed (A) and open (B) mouth positions and coronal T1, T2 (C, D) revealed anteriorly displaced deformed "amorphous" articular disc (arrow) with no reduction in open mouth position associated with medial displacement in coronal images (rotational anteromedial displacement). b (A-D) Dynamic MRI study of right TMJ revealed anteriorly displaced articular disc (arrow) with no reduction on opening of the mouth associated with limited condylar translation

- Each static study was evaluated for the following criteria in both joints (examined 80 joints):

- Articular disc shape in closed mouth position.

- Disc position: relation of the disc to the mandibular condyle as illustrated in (Fig. 1).

- Disc mobility and stuck disc (immobile disc in relation to the glenoid fossa and the articular eminence in both open and closed positions).

- Condylar translation; assessed in PD sagittal oblique images in open mouth position.

- Joint effusion and degenerative changes.

- Retrodiscal tissue; ruptured or pseudodisc.

- Each dynamic MRI study was evaluated for disc position, disc mobility, and condylar translation in both joints (examined 80 joints).

- Image quality was evaluated as regards to anatomic visibility and detection rate of TMJ structures (articular disc and condylar head) and presence or absence of motion artifact. Anatomic visibility of both articular disc and condylar head was divided into three grades: good, fair, and poor.

- Articular disc: Good-both anterior and posterior bands are seen of normal hypointense signal and thickness with visualization during whole range of movement (by dynamic MRI) and in closed and open positions by static MRI. Fair-only $50 \%$ of the disc is seen during whole range of movement (by dynamic MRI) and in closed and open positions by static MRI or the disc is seen in closed position but not detected on opening of the mouth. Poor - whole disc could not be visualized.

- Condylar head: Good-clear contour of the head with visualization during whole range of movement (by dynamic MRI) and in closed and open positions by static MRI. Fair-only $50 \%$ of the head is seen during whole range of movement (by dynamic MRI) and in closed and open positions by static MRI or the head is seen in closed position but not detected on opening of the mouth. Poor-condylar head could not be detected at all.

\section{Statistical analysis}

- The collected data were coded, processed, and analyzed using the SPSS (Statistical Package for
Social Sciences) version 22 for Windows ${ }^{\circledR}$ (IBM SPSS Inc, Chicago, IL, USA).

- Data were tested for normal distribution using the Shapiro-Wilk test. Qualitative data were represented as frequencies and relative percentages. Quantitative data were expressed as mean \pm SD (standard deviation) and range.

- Comparisons between static and dynamic MRI were performed using chi-square test.

- Probability ( $p$ value): $p$ value $<0.05$ was considered significant. $p$ value $>0.05$ was considered insignificant.

\section{Results}

This current prospective study included 40 patients with clinical diagnosis of temporomandibular joint dysfunction; 29 of them were females (72.5\%) and 11 were males (27.5\%). The age of the selected patients ranged from 18 to 66 years with a mean of 29.3 years and SD 10.68. The majority of the studied patients (25 patients) were found at the age group from 18 to 30 years $(62.5 \%)$.

All the studied patients presented clinically by temporomandibular joint pain; 36 (90\%) of them had other associated symptoms such as clicking sound in 18 patients (45\%), while limitation of movement was found in 18 patients $(45 \%)$.

Clinically, unilateral temporomandibular joint dysfunction was found in 19 patients (47.5\%) (right side was more affected than left one; $27.5 \%$ and $20 \%$, respectively), bilateral temporomandibular joint dysfunction was found in 7 patients (17.5\%), and no affection could be assessed in 14 patients (35\%).

All patients were subjected to MRI examination of the temporomandibular joint (80 joints). The normal biconcave disc shape was found in 57 joints (71.25\%) while deformed shape was found in 23 joints $(28.75 \%)$, folded in 6 joints $(7.5 \%)$, flattened in 7 joints $(8.75 \%)$, eyeglass in 5 joints $(6.25 \%)$, amorphous in 4 joints $(5 \%)$, and perforated in 1 joint (1.25\%).

Static MRI has reported 15 patients (37.5\%) with normal disc position in both joints (Fig. 2a), bilateral pathology in 6 patients (15\%), and unilateral pathology in 19 patients $(47.5 \%)$ with sensitivity of $93.3 \%$. Out of the examined 80 joints, 49 joints were normal (61.25\%), and anterior disc displacement with reduction was found in 11 joints (13.75\%) (Fig. 3a) and with no reduction in 18 


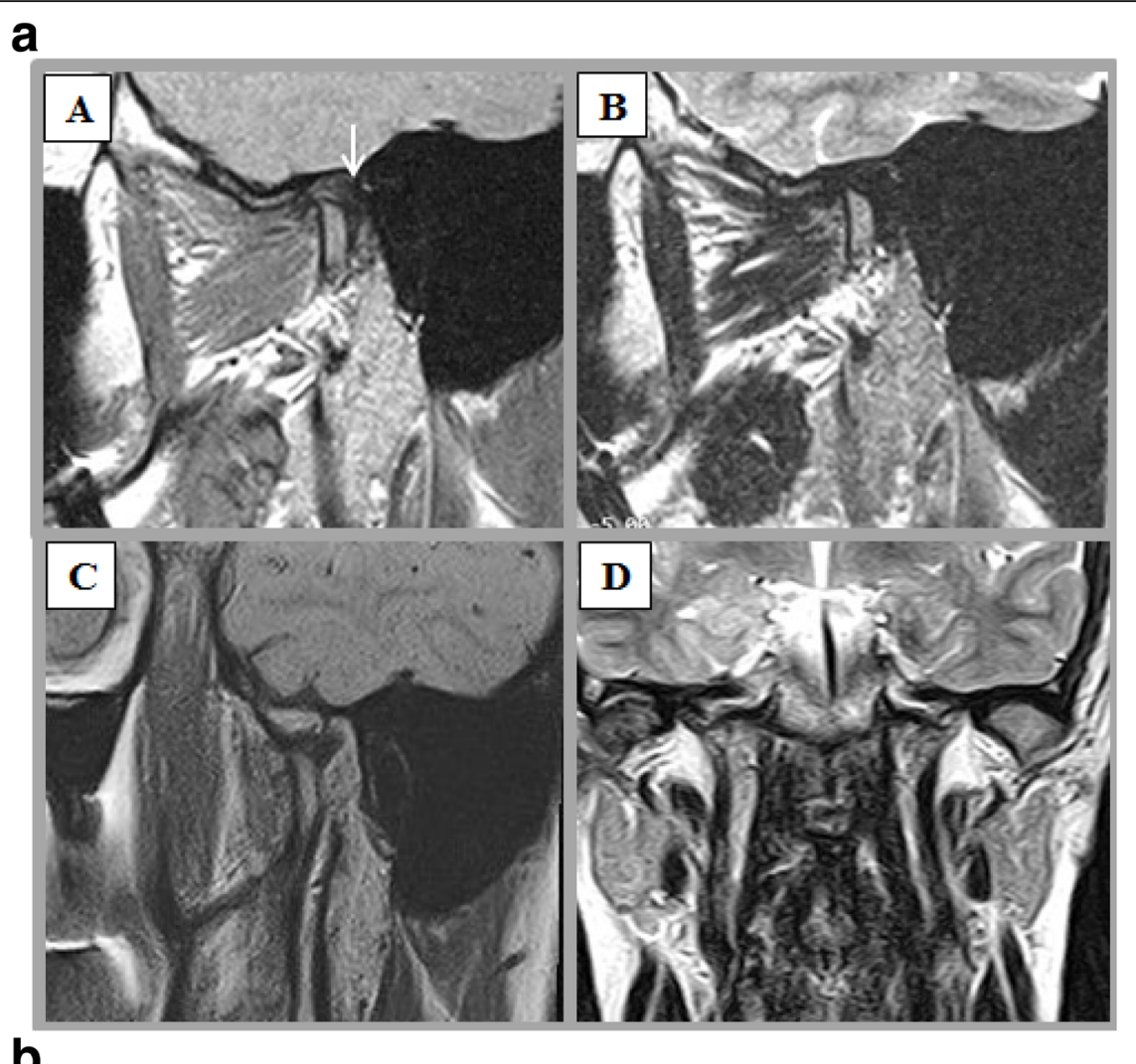

b
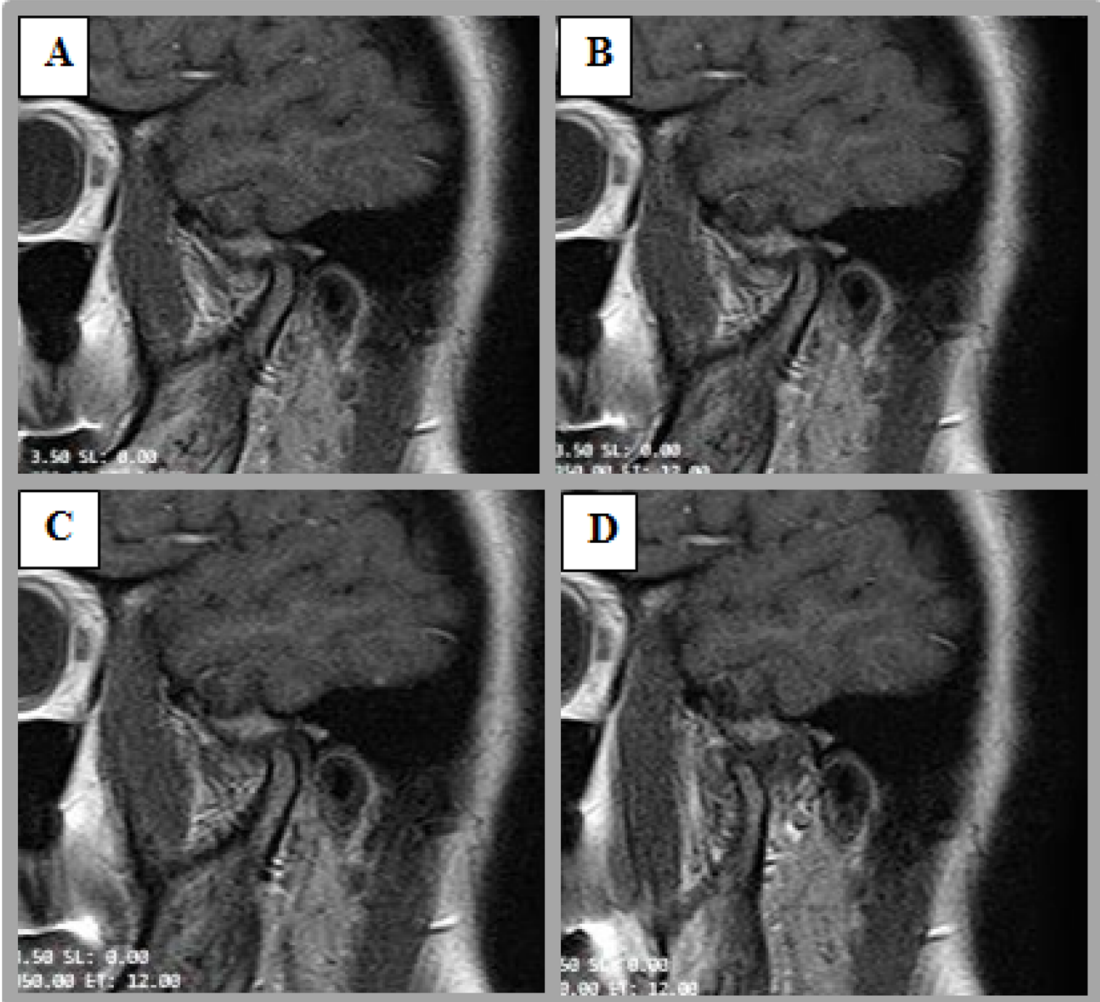

Fig. 6 (See legend on next page.) 
(See figure on previous page.)

Fig. 6 a (A-D) A 27-year-old female patient, presented clinically by left side temporomandibular joint pain and clicking. Sagittal PDWI (A) and T2WI (B) MRI of left TMJ in closed and open (C) mouth position and coronal T2 (D) revealed posterior disc dislocation with disc tissue located posterior to the condyle between posterior surface of the condyle and the post glenoid tubercle(arrow). b (A-D) Dynamic MRI study of left TMJ revealed posteriorly displaced articular disc with normal movement during mouth opening with reduction at maximum opening of the mouth (intemediate zone lies between condyle and articular eminence) associated with normal condylar translation

joints (22.5\%) (Fig. 4a). Medial displacement was detected in 4 joints (5\%) but in association with anterior displacement (rotational anteromedial displacement) (Fig. 5) while lateral displacement was detected in 1 joint $(1.25 \%)$. Posterior dislocation was diagnosed in 1 joint (1.25\%) (Fig. 6a).

Dynamic MRI reported 18 patients (45\%) with normal disc position in both joints (Fig. 2b, c), bilateral pathology in 8 patients $(20 \%)$, and unilateral pathology in 14 patients (35\%) with sensitivity of $90.9 \%$. Out of the examined 80 joints, 50 joints were normal (62.5\%), and anterior disc displacement with reduction was reported in 13 joints (16.25\%) (Fig. 3b) and with no reduction in 16 joints (20\%) (Fig. 4b). No joints were reported as medial and lateral displacement by dynamic MRI. Posterior displacement was reported in 1 joint (1.25\%) (Fig. 6b).

As regards to disc mobility, static MRI detected normal disc mobility in 78 joints (97.5\%) and stuck disc in 2 joints (2.5\%) while dynamic MRI detected normal disc mobility in 76 joints (95\%) and stuck disc in 4 joints (5\%) confirmed later by arthroscopy to be stuck disc, so in comparison to static MRI, the sensitivity of dynamic MRI in stuck disc joints is of $100 \%$ versus 50\% (Fig. 7a, b). Comparsion between static and dynamic MRI in evaluation of disc position and mobility of the examined joints is illuetrated at (Table 1).

In static MRI, condylar translation was normal in 62 joints (77.5\%), hypomobile in 17 joints (21.25\%), and hypermobile in only 1 joint (1.25\%), while by dynamic MRI, normal condylar translation was normal in 54 joints (67.5\%), hypomobile in 18 joints (22.5\%), and hypermobile in 8 joints (10\%) as described in Table 2.

Other TMJ structures (other than the articular disc) were evaluated, and patients are distributed according to presence or absence of joint effusion, degenerative changes, and evaluation of retrodiscal tissue. MRI analysis of the studied patients revealed presence of joint effusion in 16 joints (20\%) and degenerative changes in 17 joints (21.25\%). Retrodiscal tissue was evaluated, it was normal in 71 joints $(88.75 \%)$ and ruptured in 1 joint (1.25\%), and pseudodisc was found in 9 joints (11.25\%). Both rupture and pseudodisc were detected in 1 joint (1.25\%).

\section{Dynamic MRI image quality evaluation:}

Anatomic visibility and detection rate of articular disc and condylar head during movement were evaluated and compared with detection rate of static MRI as gold standard as shown in Table 3.

Static MRI could detect the articular disc well in 30 patients $(75 \%)$, fairly in 7 patients $(17.5 \%)$, and poorly in 3 patients $(7.5 \%)$, while dynamic MRI could detect the disc well in 17 patients (42.5\%), fairly in 18 patients (45\%), and poorly in 5 patients $(12.5 \%)$. Detection rate of articular disc for dynamic MRI is $87.5 \%$ versus static MRI 92.5\% ( $p$ value 0.038 ).

As regards to condylar head, static MRI could detect the condylar head well in 37 patients $(92.5 \%)$, fairly in 3 patients $(7.5 \%)$, and poorly in 0 patients, while dynamic MRI could detect the condylar head well in 30 patients (75\%), fairly in 9 patients $(22.5 \%)$, and poorly in 1 patient (2.5\%). Detection rate of condylar head for dynamic MRI is $97.5 \%$ versus static MRI $100 \%$ ( $p$ value 0.012 ).

The second parameter for evaluating the image quality is motion artifact; it is detected in 12 patients (30\%) in dynamic MRI versus 13 patients (32.5\%) in static MRI as shown in Table 4 ( $p$ value 0.032 ).

\section{Discussion}

The most prevalent cause of temporomandibular joint (TMJ) dysfunction is internal derangement, which is defined as an abnormal relationship of the disc to the condyle. Disc displacement is the most popular form of TMJ dysfunction [10, 11].

Temporomandibular joint dysfunction was previously assessed by arthrography using computed tomography as a standard method as it provides an accurate visualization of soft and hard tissue and good evaluation of the bony structures of TMJ. However, this painful invasive procedure carries a risk of iatrogenic disc perforation and damage of facial nerve, suffers from difficulties in patient positioning, exposes him to radiation, and depends on examiner's skill [12].

It is generally preceded using routine pulse sequences with acquisition of static sagittal and coronal images during mouth opening and closing. Although static views are derived before or after disc reduction and cannot cover the proper physiologic process, static MRI was the gold standard in diagnosing TMJ dysfunction $[9,13]$.

At present, dynamic MRI is introduced in the set of dynamic assessments of joint function. In the current study, 40 patients with temporomandibular joint pain did static and dynamic MRI examinations of both temporomandibular joints. 


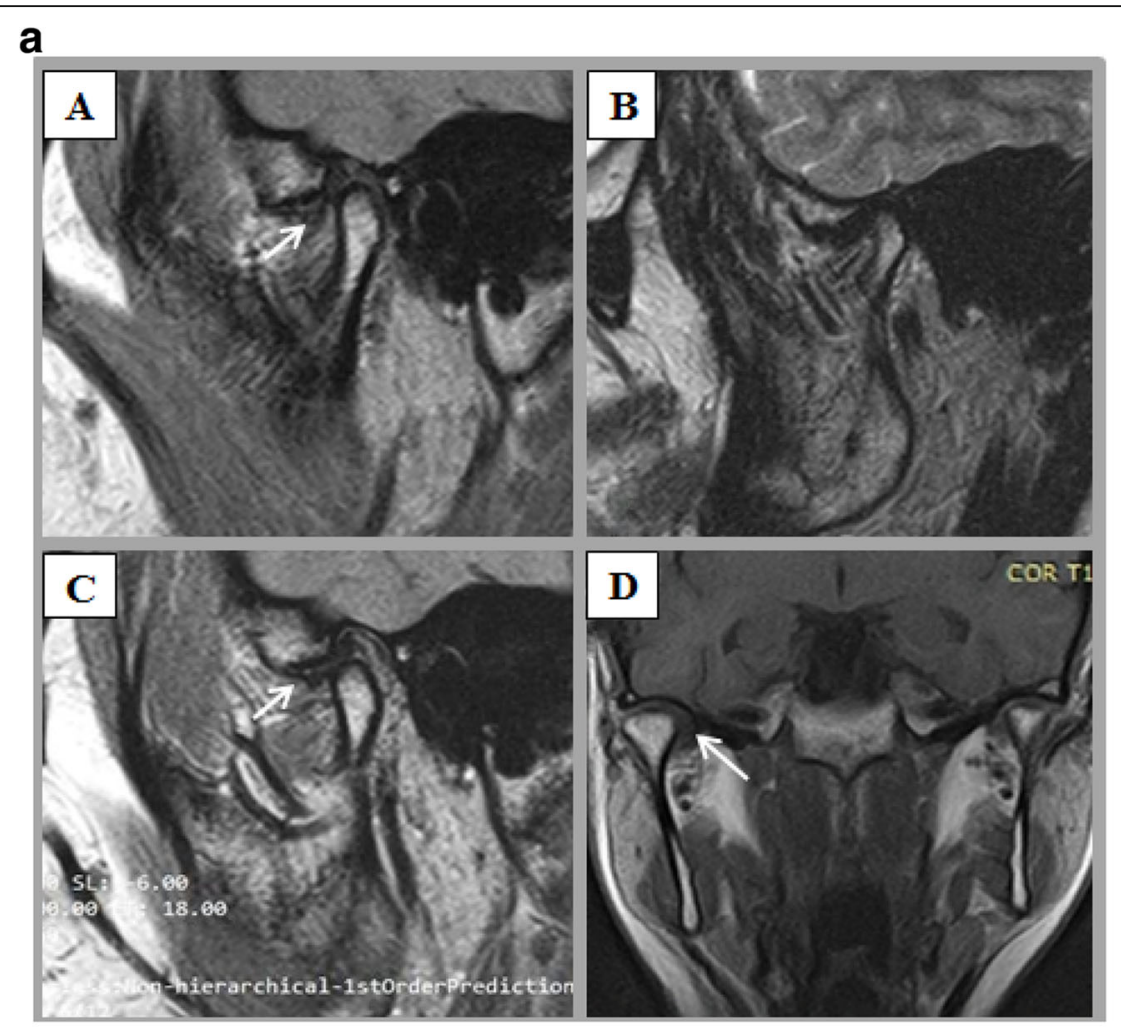

b
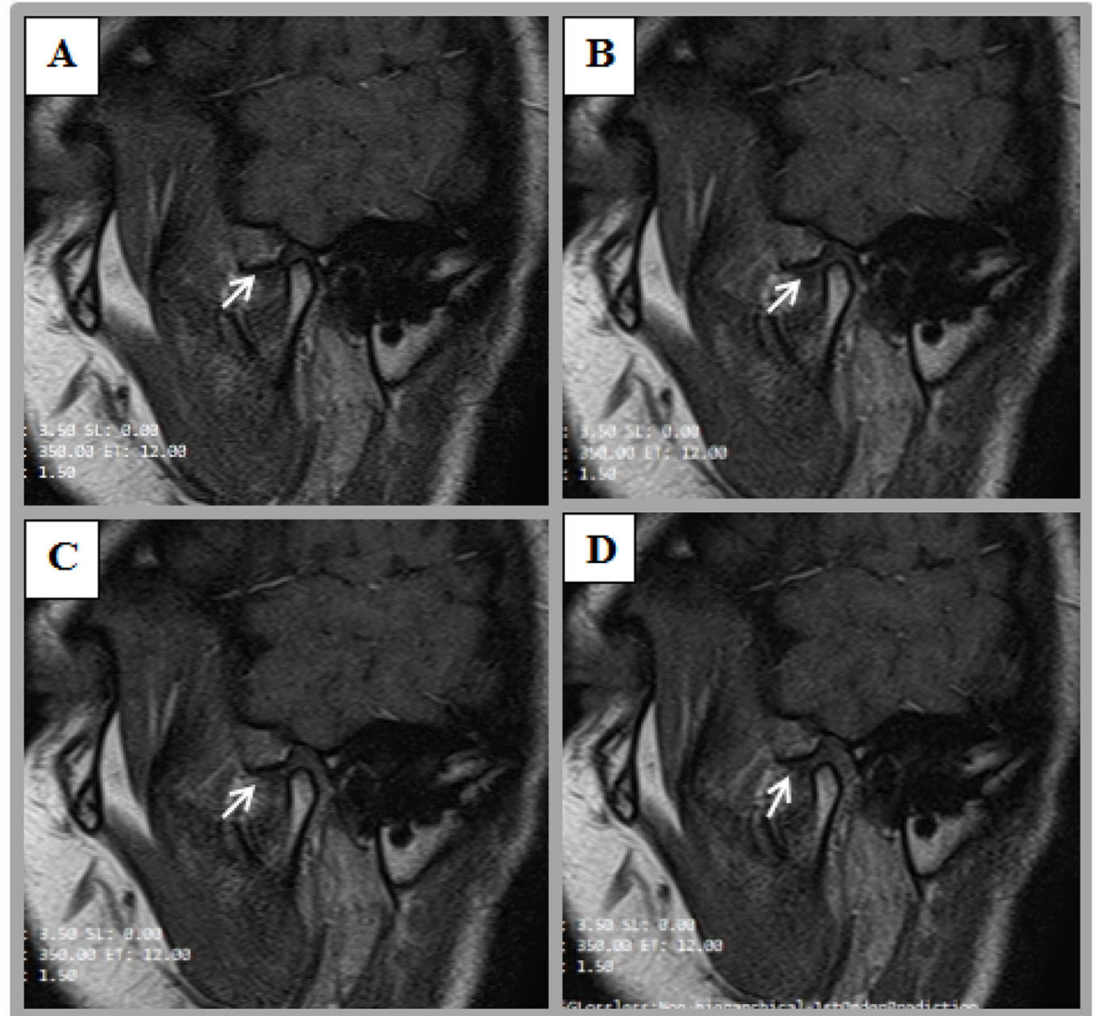

Fig. 7 (See legend on next page.) 
(See figure on previous page.)

Fig. 7 a (A-D) A 29-year-old male patient, presented clinically by TMJ pain and limitation of movement on the right side. Sagittal PDWI (A) and T2WI (B) MRI of right TMJ in closed and open (C) mouth positions and coronal T1 (D) revealed normal position of articular disc (arrow) in closed mouth position with no change in its position in open mouth position (stuck disc) associated with slight medial displacement in coronal image. b (A-D) Dynamic MRI study of right TMJ revealed normal disc position in closed mouth position with no changes in position on opening of the mouth associated with hypomobile condylar translation

Disc displacement is more common in females than males (3:1) and commonly appears in the second to fourth decades of life [14]. Among those 40 patients, $72.5 \%$ were females and $27.5 \%$ were males with ratio 2.6 :

1. This female predominance agrees with Vieira-Queiroz et al. [15] who performed their study on 185 patients; $78.9 \%$ of them were females and $21.1 \%$ were males with ratio 3.7:1.

A wide range of age group was included in this study, ranging from 18 to 66 years with a mean of 29.3 years and $\mathrm{SD} \pm 10.68$. TMJ dysfunction affects young ages (between 20 and 40 years) more than old age patients (more than 60 years). Dupuy-Bonafé et al. [16] performed their study on 40 patients; their age ranged from 21 to 59 with median 25.8. Also, in the study of Vogl et al. [17], there were 794 patients, and their age ranged from 14 to 83 years with mean 38.7 and $\mathrm{SD} \pm 16$.

Disc position was evaluated in our study by both static and dynamic MRIs with sensitivity of $93.93 \%$ versus 90.9\% ( $p$ value $<0.001$ ). The prevalence of disc displacement with reduction (DDwR) in patients affected by temporomandibular disorders ranges from 24 to $47 \%$ while that of disc displacement without reduction (DDwNR) from 11 to 26\% [18].

Anterior disc displacement was the most common finding among examined joints, and anterior disc displacement

Table 1 Comparison between static and dynamic MRI in evaluation of disc position and mobility in the examined joints $(n=80)$

\begin{tabular}{|c|c|c|c|c|c|c|}
\hline & & \multicolumn{2}{|c|}{ Static MRI } & \multicolumn{2}{|c|}{ Dynamic MRI } & \multirow[t]{2}{*}{$p$} \\
\hline & & No. & $\%$ & No. & $\%$ & \\
\hline \multirow[t]{6}{*}{ Disc position } & Normal & 49 & 61.25 & 50 & 62.5 & \\
\hline & ADDwR & 11 & 13.75 & 13 & 16.25 & $<0.001^{*}$ \\
\hline & ADDwNR & 18 & 22.5 & 16 & 20.0 & $<0.001^{*}$ \\
\hline & Medial & 4 & 5.0 & 0 & 0.0 & - \\
\hline & Lateral & 1 & 1.25 & 0 & 0.0 & - \\
\hline & Posterior & 1 & 1.25 & 1 & 1.25 & $0.025^{*}$ \\
\hline \multirow[t]{2}{*}{ Disc mobility } & Normal mobility & 78 & 97.5 & 76 & 95.0 & $0.008^{*}$ \\
\hline & Stuck disc & 2 & 2.5 & 4 & 5.0 & \\
\hline
\end{tabular}

Four joints had combined medial and anterior displacement (rotational anteromedial displacement) as reported by static MRI

$A D D w R$ anterior disc displacement with reduction, $A D D w N R$ anterior disc displacement with

no reduction, $p p$ value for chi-square test for association between static MRI and dynamic MRI

*Statistically significant at $p \leq 0.05$ with reduction (ADDwR) was found in 11 joints $(13.75 \%$ of all joints) and with no reduction in 18 of them (22.5\% of all joints) by static MRI. Dynamic MRI reported ADDwR in 13 joints (16.25\%) and ADDwNR in 16 joints (20\%). Whyte et al. [10] reported in their study on 144 patients $(288$ joints) 50 normally positioned discs and 226 joints with anterior displacement: 136 joints were reduced and 90 joints are non-reduced. Zhang et al. [9] found 10 cases (16.7\%) out of 30 patients with ADDwR, and one case (1.7\%) with ADDwNR.

Medial and lateral disc displacements were detected in static MRI only. This is because the dynamic MRI with its sagittal views has limitation in the evaluation of sideway displacements as it is well evaluated in the coronal plane. The disc crosses over one of the sagittal planes tangential to one of the condylar poles without an anterior component. The empty fossa sign seen in the sagittal images is an indication of a medial or lateral disc displacement [19].

Posterior disc displacement is a rare TMJ disorder. It was overlooked in the past because of a lack of welldefined imaging characteristics, and its diagnosis depended mainly on clinical signs. A definitive diagnosis requires magnetic resonance imaging (MRI) [20]. Posterior dislocation was diagnosed in 1 joint by both static and dynamic MRIs in our study. The meta-analysis study of Afroz et al. [21] reported the overall prevalence of posterior disc displacement (PDD) for the number of joints affected was $0.7 \%$ and for the number of patients affected was $0.9 \%$.

A stuck or fixed TMJ disc is the disc that remained fixed in position relative to the glenoid fossa and the articular eminence in both closed and opened mouth projections. It is caused mainly by joint adhesion which cannot be detected by MRI, and it is detected by direct

Table 2 Comparison between static and dynamic MRI in evaluation of condylar translation in the examined joints $(n=80)$

\begin{tabular}{lllllll}
\hline \multirow{2}{*}{$\begin{array}{l}\text { Condylar } \\
\text { translation }\end{array}$} & \multicolumn{2}{l}{ Static MRI } & & \multicolumn{2}{c}{ Dynamic MRI } & \multirow{2}{*}{$\boldsymbol{p}$} \\
\cline { 2 - 3 } & No. & $\%$ & & No. & $\%$ & \\
\hline Normal & 62 & 77.5 & & 54 & 67.5 & $<0.001^{*}$ \\
Hypomobile & 17 & 21.25 & & 18 & 22.5 & \\
Hypermobile & 1 & 1.25 & & 8 & 10.0 & \\
\hline
\end{tabular}

$p: p$ value for chi-square test for association between static MRI and dynamic MRI

*Statistically significant at $p \leq 0.05$ 
Table 3 Anatomic visibility and detection rate of both articular discs and condylar heads in static and dynamic MRI in the studied patients $(n=40)$

\begin{tabular}{|c|c|c|c|c|c|c|}
\hline & & \multicolumn{2}{|c|}{ Static MRI } & \multicolumn{2}{|c|}{ Dynamic MRI } & \multirow[t]{2}{*}{$p$} \\
\hline & & No. & $\%$ & No. & $\%$ & \\
\hline \multirow[t]{3}{*}{ Disc detection } & Good & 30 & 75.0 & 17 & 42.5 & $0.038^{*}$ \\
\hline & Fair & 7 & 17.5 & 18 & 45.0 & \\
\hline & Poor & 3 & 7.5 & 5 & 12.5 & \\
\hline \multirow[t]{3}{*}{ Condylar head detection } & Good & 37 & 92.5 & 30 & 75.0 & $0.012^{*}$ \\
\hline & Fair & 3 & 7.5 & 9 & 22.5 & \\
\hline & Poor & 0 & 0.0 & 1 & 2.5 & \\
\hline
\end{tabular}

$p: p$ value for chi-square test for association between static MRI and dynamic MRI

*Statistically significant at $p \leq 0.05$

visualization or arthroscopy but, at this time, can detect disc immobility as an indicator of disc adhesion [22].

In the current study, static MRI reported normal disc mobility in 78 joints and stuck disc in 2 joints, while dynamic MRI reported normal disc mobility in 76 joints and stuck disc in 4 joints $(p$ value $=0.008)$. Arthroscopy was done later on as the patients were complaining seriously. Arthroscopy confirms that they had stuck discs. So, dynamic MRI may have higher accuracy in diagnosis of stuck disc than static MRI.

Eberhard et al. [11] evaluated disc mobility in 40 patients with TMJ dysfunction symptoms by dynamic MRI which revealed 2 cases of stuck disc (disc adhesions). Also, in the study of Amin et al. [23] on 28 patients, dynamic MRI reported 13 patients with normal disc mobility, 12 patients with limited asynchronous mobility, and 3 patients with stuck disc.

As regards to condylar translation, it was classified as either normal (apex of condyle meets the apex of articular eminence), hypomobile (condyle not reaching the apex of articular eminence), or hypermobile (condyle reaches beyond the articular eminence). Static MRI reported 62 joints with normal condylar translation, 17 joints with hypomobile condylar translation, and 1 joint with hypermobile condyles, while dynamic MRI reported 54 joints with normal condylar translation, 18 joints with hypomobile condylar translation, and 8 joints with hypermobile condyles $(p$ value $<0.001)$. The ability of

Table 4 Motion artifact in static and dynamic MRI in the studied patients $(n=40)$

\begin{tabular}{lllllll}
\hline \multirow{2}{*}{$\begin{array}{l}\text { Motion } \\
\text { artifact }\end{array}$} & \multicolumn{2}{l}{ Static MRI } & & \multicolumn{2}{c}{ Dynamic MRI } & \multirow{2}{c}{$\boldsymbol{p}$} \\
\cline { 2 - 3 } & No. & $\%$ & & No. & $\%$ & \\
\hline No & 27 & 67.5 & & 28 & 70.0 & $0.032^{*}$ \\
Yes & 13 & 32.5 & & 12 & 30.0 & \\
\hline
\end{tabular}

$p: p$ value for chi-square test for association between static MRI and dynamic MRI

*Statistically significant at $p \leq 0.05$ dynamic MRI to detect hypermobile condyles with rate higher than static MRI may be attributed to that dynamic MRI is capable of imaging the full range of motion of condylar translation due to the use of voluntary motion, rather than a fixed intraoral device to open the mouth.

Beer et al. [24] demonstrated good correlation in range of motion between dynamic MRI and static MRI $(p$ value $=0.001)$ that is confirmed by axiography. These results do not go with the study of Wang et al. [25] who reported that dynamic MRI detects normal motion more than static MRI while static detects limited mobility (30.4\%) more than dynamic MRI (17.7\%).

Disc displacements are associated with pathological changes such as joint effusion, degenerative changes, and changes in retrodiscal tissue [26]. In our study, all of these changes were associated with different types of disc displacement. Out of examined 9 joints with retrodiscal tissue changes, 7 joints had ADDwNR and 2 joints had ADDwR. Higuchi et al. [26] reported in their study that joint effusion was detected in 63 patients (49\%) of 129 patients presented with TMJ pain. Also in the study of Campos et al. [27], degenerative changes were detected in 104 patients; 76 of them diagnosed to have disc displacement. Hasan et al. [3] reported in their study that morphological changes of retrodiscal layers were most frequently observed in ADDwNR (88.9\%) than ADDwR.

Dynamic MRI was evaluated as a diagnostic tool by comparing it to the static MRI by two parameters; the first is by findings and ability to diagnose different types of disc displacement and the second by evaluating the quality of the image as regards to anatomic visibility of important TMJ structures (articular disc and mandibular condyle) and presence of motion artifact.

As regards to quality of the images acquired by dynamic MRI, detection of disc and condylar head was rated as whether good, fair, or poor and compared to the detection of static MRI. Disc detection rate by dynamic sequence was $87.5 \%$ versus $92.5 \%$ by static MRI ( $p$ value 0.038 ). Condylar head detection rate was $97.5 \%$ for dynamic MRI versus $100 \%$ for static MRI ( $p$ value 0.012). Shimazaki et al. [28] reported in their study high detection rate of articular disc $83 \%$ and of condylar head $95 \%$ by dynamic sequence. Poor detection of disc in some cases may be attributed to either degenerative changes causing thinning of the disc and increasing its signal or sideway displacement of the disc shifting the disc away from imaging plane [28].

One of the challenges of dynamic MRI is to acquire images of moving object with minimal motion artifact [28]. In our study, dynamic sequence is associated with decreased motion artifacts, despite ongoing motion of the joint. Motion artifact was detected in 12 patients (30\% of 
patients) versus 13 patients (32.5\% of patients) ( $p$ value 0.032 ). This agrees with the study of Wang et al. [25] who reported that static examinations were rated as having motion artifact $(19.6 \%$ versus $6.9 \%, p .016)$.

Regarding to the final outcome, we realized that dynamic MRI facilitated visualization of the articular disc during the entire course of jaw opening and closing as well as the dynamics of internal derangement and assessment of disc condyle relationship in a short time compared to static MRI. It is better in evaluating disc mobility than static MRI, but static MRI is better in evaluating side by side displacement which is an important limitation of dynamic MRI. Detection rate of articular disc and condylar head is lower in dynamic than static MRI which needs further studies to improve it.

Amin et al. [23] reported that MRI of TMJ using its dynamic and static sequences is more sensitive than arthroscopy in evaluating disc position and mobility and recommend using MRI as a primary modality for diagnosis in suspected internal derangement.

The main limitation in our study is longer time of examination, the lower detection rate of articular disc by dynamic MRI compared to static MRI that needs further studies for improving it.

\section{Conclusion}

In conclusion, dynamic MRI sequences cannot be used as an alternative to static sequences in the evaluation of internal derangement disorders of TMJ; it should be used as a routine MRI examination complementary to static sequences in order to diagnose different types of disc displacement and achieving the best image quality.

\section{Abbreviations}

ADDwR: Anterior disc displacement with reduction; ADDwNR: Anterior disc displacement with no reduction; TMD: Temporomandibular joint disorders; TMJ: Temporomandibular joint

\section{Acknowledgements}

To all the participants for their cooperation and patience.

\section{Authors' contributions}

All authors read and approved the final manuscript for submission. $\mathrm{MH}$ suggested the research idea, ensured the original figures and data in the work, minimized the obstacles to the team of work, correlated the study concept and design, and had the major role in analysis. ZS collected the data in all stages of the manuscript and performed the data analysis. OA supervised the study with significant contribution to the design of the methodology, manuscript revision, and preparation. HA correlated the clinical data of patients and matched it with the findings, and drafted and revised the work.

\section{Funding}

No funding. Not applicable for this section.

\section{Availability of data and materials}

The authors confirm that all data supporting the finding of the study are available within the article and the raw data and data supporting the findings were generated and available at the corresponding author on request.

\section{Ethics approval and consent to participate}

Informed written consents were taken from the patients and healthy volunteers; the study was approved by the ethical committee of Tanta University Hospital, Faculty of Medicine. Committee's reference number: 32840/1/19.

\section{Consent for publication}

All participants included in the research gave written consent to publish the data included in the study.

\section{Competing interests}

The authors declare that they have no competing interests.

Received: 22 September 2020 Accepted: 16 December 2020 Published online: 08 January 2021

\section{References}

1. Sun Q, Dong M, Tao X et al (2015) Dynamic MR imaging of temporomandibular joint: an initial assessment with fast imaging employing steady-state acquisition sequence. Magnetic Resonance Imaging 33(3):270275

2. Krohn S, Joseph AA, Voit D, et al (2019) Multi-slice real-time MRi of temporomandibular joint dynamics. Dentomaxillofacial Radiol 48(1): 20180162

3. Hasan NM, Abdelrahman TE (2014) MRI evaluation of TMJ internal derangement: degree of anterior disc displacement correlated with other TMJ soft tissue and osseous abnormalities. Egypt J Radiol Nuclear Med 45(3):735-744

4. Petscavage-Thomas JM, Walker EA (2014) Unlocking the jaw: advanced imaging of the temporomandibular joint. Am J Roentgenol 203(5):10471058

5. (Ertem SY, Konarılı FN, Ercan K (2019) Does incidence of temporomandibular disc displacement with and without reduction show similarity according to MRI results? J Maxillofacialnad Oral Surgery 19:603-608

6. Yen P, Katzberg RW, Buonocore MH et al (2013) Dynamic MR imaging of the temporomandibular joint using a balanced steady-state free precession sequence at 3T. Am J Neuroradiol 34(3):24-26

7. Bag AK (2014) Imaging of the temporomandibular joint: an update. World J Radiol 6(8):567

8. Marotti M (2010) Imaging of temporomandibular joint disorders. Rad 507. Med Sci 34:135-148

9. Zhang S, Gersdorff N, Frahm J (2011) Real-time magnetic resonance imaging of temporomandibular joint dynamics. Open Med Imaging J 5:1-9

10. Whyte AM, McNamara D, Rosenberg I et al (2019) Magnetic resonance imaging in the evaluation of temporomandibular joint disc displacement-a review of 144 cases. Int J Oral Maxillofacial Surg 35(8):696-703

11. Eberhard D, Bantleon H, Steger W (2000) Functional magnetic resonance imaging of temporomandibular joint disorders. Eur J Orthodontics 22:489497

12. Krohna S, Gersdorffa N, Wassmanna T et al (2016) Real-time MRI of the temporomandibular joint at 15 frames per second - a feasibility study. Eur J Radiol 85(2016):2225-2230

13. Alonso M, Gamba T, Lopes $S$ et al (2014) Magnetic resonance imaging of the temporomandibular joint acquired using different parameters. J Morphol Sci 31(2):103-109

14. Progress M, Scrivani SJ, Keith DA et al (2008) Temporomandibular disorders. New England J Med 359(25):2693-2705

15. Vieira-Queiroz I, Gomes Torres MG, Oliveira-Santos D et al (2013) Biometric parameters of the temporomandibular joint and association with disc displacement and pain: a magnetic resonance imaging study. Int J Oral Maxillofac Surg 42(6):765-770

16. Dupuy-Bonafé I, Picot MC, Maldonado IL et al (2012) Internal derangement of the temporomandibular joint: is there still a place for ultrasound? Oral Surg Oral Med Oral Pathol Oral Radiol 113(6):832-840

17. Vogl TJ, Lauer HC, Lehnert T et al (2016) The value of MRI in patients with temporomandibular joint dysfunction: correlation of MRI and clinical findings. Eur J Radiol 85(4):714-719

18. Evans MRB. (2020) MRI of the clinical medicine temporomandibular covertemplate joint. (Tanteri TRT, ed.). Springer Nature Switzerland AG. 
19. Orhan K (2019) Magnetic resonance imaging of TMJ. In: Rozylo-Kalinowska, Ingrid, Orhan, Kaan (Eds.). Imaging of the temporomandibular joint.; Springer Nature Switzerland AG (10): 155-204 .

20. Chossegros C, Cheynet F, Guyot L, et al (2001) Posterior disc displacement of the TMJ: MRI evidence in two cases Cranio 19 (4):289-93.

21. Afroz S, Naritani M, Hosoki H, et al (2018) Prevalence of posterior disc displacement of the temporomandibular joint in patients with temporomandibular disorders: systematic review and meta-analyses. J Oral Facial Pain Headache 32(3):277-286

22. Zhang SY, Yang C, Chen MJ et al (2009) Magnetic resonance imaging in the diagnosis of intra-articular adhesions of the temporomandibular joint. Brit J Oral Maxillofacial Surg 47(5):389-392

23. Amin MF, Hassan AM, Barakat KI (2012) The accuracy of dynamic magnetic resonance imaging in evaluation of internal derangement of the temporomandibular joint; comparison with arthroscopic findings. Egypt J Radiol Nuclear Med 43(3):429-436

24. Beer A, Kolk A, Neff A, et al (2004) Cine MRI of the temporomandibular joint in comparison to static MRI and axiography (Cine-MRT des Kiefergelenks im Vergleich zur konventionellen MRT und Achsiographie). Fortschr R"ntgenstr 176: $506-512$.

25. Wang EY, Mulholland TP, Pramanik BK et al (2007) Dynamic sagittal halfFourier acquired single-shot turbo spin-echo MR imaging of the temporomandibular joint: initial experience and comparison with sagittal oblique proton-attenuation images. Am J Neuroradiol 28(6):1126-1132

26. Higuchi K, Chiba M, Sai Y et al (2019) Relationship between temporomandibular joint pain and magnetic resonance imaging findings in patients with temporomandibular joint disorders. Int J Oral Maxillofacial Surg 30(2):118-122

27. Campos M, Campos P, Cangussu M et al (2008) Analysis of magnetic resonance imaging characteristics and pain in temporomandibular joints with and without degenerative changes of the condyle. Int J Oral Maxillofac Surg 37(6):529-534

28. Shimazaki Y, Saito K, Matsukawa S et al (2007) Image quality using dynamic MR imaging of the temporomandibular joint with true-FISP sequence. Magnetic Resonance Med Sci 6(1):15-20

\section{Publisher's Note}

Springer Nature remains neutral with regard to jurisdictional claims in published maps and institutional affiliations.

\section{Submit your manuscript to a SpringerOpen ${ }^{\circ}$ journal and benefit from:}

- Convenient online submission

- Rigorous peer review

- Open access: articles freely available online

- High visibility within the field

- Retaining the copyright to your article

Submit your next manuscript at $\boldsymbol{\nabla}$ springeropen.com 